\title{
Efficient beamforming and spectral efficiency maximization in a joint transmission system using an adaptive particle swarm optimization algorithm
}

\begin{abstract}
Next-generation cellular networks and beyond are expected to adopt a frequency reuse factor of one to support high spectral efficiency. Consequently, inter-cell interference (ICI) represents a serious issue among neighbouring cells, especially for cell-edge users. In addressing this, joint transmission (JT) represents one of the most sophisticated techniques for mitigating ICI stemming from implementing a frequency reuse factor of one. Moreover, JT also converts the interfering signals into useful signals to improve the spectral efficiency of the system. However, JT produces enormous overhead on both the feedback and backhaul interfaces; thus, partial JT was proposed as a trade-off between signaling demand and increased spectral efficiency. Maintaining an efficient beamforming (BF) matrix based on a sparse aggregated channel matrix is a challenging issue with regard to linear BF techniques such as zero-forcing (ZF). This is mainly because ZF BF can only invert a well-conditioned matrix. Therefore, an adaptive particle swarm optimization (APSO) is included in this paper and used to present an efficient beamformer that achieves equivalent backhaul reduction and high spectral efficiency. Moreover, addressing the lack-of-diversity issue in basic particle swarm optimization (BPSO) is a primary concern of this work. The beamformer obtained with the objective function of sum rate maximization achieves a spectral efficiency of $17.24 \%$ compared to BPSO BF.
\end{abstract}

Keyword: Joint transmission; Interference avoidance; Beamforming; Particle swarm; Zeroforcing 